\title{
Use of Neural Networks in Sounding Rocket Trajectory Reconstitution
}

\author{
Alexandre N. Barbosa ${ }^{1}$, José Demisio S. da Silva ${ }^{2}$ \\ ${ }^{1}$ Master's Program in Applied Computing - CAP \\ ${ }^{2}$ Laboratory for Computing and Applied Mathematics - LAC \\ Brazilian National Institute for Space Research - INPE \\ C. Postal 515 - 12245-970 - São José dos Campos - SP \\ BRAZIL \\ E-mail: n.barbosa@iae.cta.br, demisio@lac.inpe.br
}

Keywords: neural networks, trajectory reconstitution, sounding rocket.

A rocket launch assignment takes into account three main aspects. The first one is concerned to the payload experiments. The second one is on the rocket which meets the mission requirements. The third one is on the launch infra-structure that encompasses the systems to track the rocket, such as the telemetry and radar systems. In Brazil, the priority is the radar. There exists at least two radar systems, one of proximity and another one of accuracy. Both provide the instantaneous rocket position and velocity. They should carry out without halts, but tracking interruptions could occur due to atmospheric conditions, malfunctions and so on. Such problem happened with the first flight of the Brazilian rocket VSB-30, during its launch operation in Alcantara Launch Center (CLA), in October 2004. When the tracking vanishes simultaneously in both radars, they are guided by the information of the nominal trajectory, in order to retry the tracking.

After the launching, it starts the post-flight analysis. The Flight Dynamics Subdivision of the Space and Aeronautics Institute in Brazil takes part in studying the rocket trajectory and performing its post-flight analysis. In order to carry out such analysis, computational applications are employed. Among those applications, it is the ROSI, Rocket Simulation, for trajectory calculation. If the actual trajectory is not quite tracked by the radars, it is performed its reconstitution using the ROSI software by updating its input data with actual flight conditions.

This paper treats with a typical problem of occlusion. For the time that the radars have been blinded, the nominal characteristics of the flight are not realized in the actual trajectory. Therefore, the ROSI software is employed to reconstitute such characteristics. Although the current method to reconstitute the trajectory is probably the most accurate, it takes too much effort to updating the input data of the ROSI. Besides, the complexity of the reconstitution using the ROSI increases when propelled phases and atmosphere are taken into account of the occluded stream of the actual trajectory.

Searching by an alternative method, this paper introduces the use of neural networks, in a preliminary approach, in order to reconstitute the trajectory of a sounding rocket, instead of using the ROSI software. The basic idea is to calculate the difference from both nominal and actual trajectories, and reconstitute the occluded nominal characteristics, that are not realized in the actual trajectory, by using a linear interpolation on the difference between them. After that, a multilayer perceptron is trained to map the reconstituted patterns of the actual trajectory.

Finally, for analysis of the proposed method, occlusions are simulated on a quite covered flight, such as the second one of the VSB-30, in such way that it is possible to calculate de error with respect to the omitted part of the actual trajectory, in order to validate de method and study the cases for what it diverges. In addition to this, an enhanced approach is briefly introduced for further studies.

\section{REFERENCES}

1. Haykin, S. (1999), Neural Networks: A Comprehensive Foundation, 2. Ed, Prentice Hall.

2. Hecht-Nielsen, R. (1990), Neurocomputing, Addison-Wesley.

3. Kramer, H.J., Craubner, A. and Ziegltrum, W (1976), ROSI Rocket Simulation, DFVLR TN 12/76. 